Proceedings

\title{
SERS Active Periodic 3D Structure for Trapping and High Sensitive Molecular Analysis of Particles or Cells ${ }^{\dagger}$
}

\author{
István Rigó ${ }^{1}$, Miklós Veres ${ }^{1}$ and Péter Fürjes ${ }^{2, *}$ \\ 1 Institute for Solid State Physics and Optics, Wigner Research Centre for Physics, HAS, Budapest, Hungary; \\ rigo.istvan@wigner.mta.hu (I.R.); veres.miklos@wigner.mta.hu (M.V.) \\ 2 Institute of Technical Physics and Materials Science, Centre for Energy Research, HAS, Budapest, Hungary \\ * Correspondence: furjes@mfa.kfki.hu; Tel.: +36-1-392-2222 \\ + Presented at the Eurosensors 2017 Conference, Paris, France, 3-6 September 2017.
}

Published: 8 August 2017

\begin{abstract}
In this work the highly sensitive molecule recognition performance of a specially designed surface enhanced Raman scattering (SERS) substrate was demonstrated. The general inverse pyramid structures were modified to be able to perform as cavities in perforated membrane applicable for particle and cell filtering, sorting and trapping. In the voids of the gold covered substrate size compatible particles functionalised by different molecules were trapped, their SERS signal was detected and the different molecules were recognised.
\end{abstract}

Keywords: Raman Spectroscopy; SERS; periodic 3D structure; particle trapping

\section{Introduction}

SERS [1] evolves in close vicinity of metallic nanostructures where the interaction between the electromagnetic field of photons and the surface plasmons results in several orders of magnitude enhancement of the Raman signal. This effect extremely improves the sensitivity of Raman spectroscopy achieving the limit of molecule detection in attomolar $\left(10^{-18} \mathrm{M}\right)$ concentrations [2]. According to our approach a special size fitted SERS active substrate was prepared to be applicable for particle and cell entrapment and highly sensitive detection of molecules immobilized on their surface.

\section{Materials and Methods}

Arrays of periodic perforated inverse pyramids were prepared on polished SOI (silicon-oninsulator) wafers. The 3D structure was shaped by alkaline etching from the front side in the $2 \mu \mathrm{m}$ thick device layer of the wafer by using the appropriate masking pattern. Periodic vertical tapered microchannels (see Figure 1) were formed by etching away the back-side handler silicon and buried oxide and coated with a $50 \mathrm{~nm}$ thick evaporated layer of gold. The resulted geometry was used simultaneously as an array of particle traps and a SERS substrate.

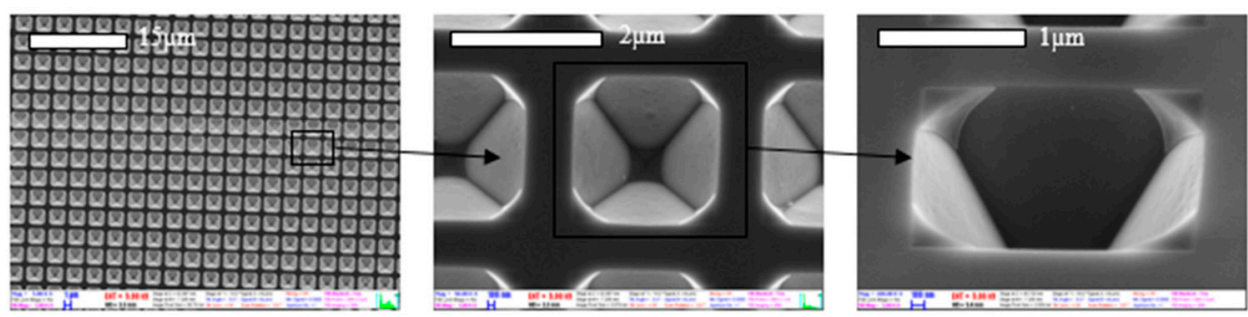

Figure 1. SEM view of the periodic perforated SERS substrate for particle and cell entrapment. 


\section{Results}

Raman spectra of benzophenone were recorded (by a Renishaw 1000 micro-Raman spectrometer with Leica DM/LM microscope using 1 micron laser spot size on the sample and $785 \mathrm{~nm}$ excitation wavelength) on flat silicon and the structured gold surface. The significant amplification by the SERS substrate is presented in Figure 2.

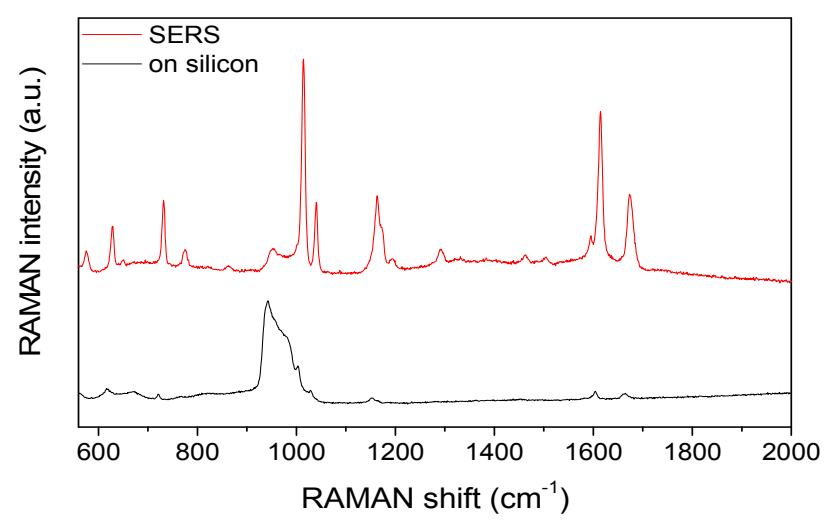

Figure 2. Comparison of the Raman signals of benzophenone detected on silicon and the 3D structured surface. The broad band in the $900-1000 \mathrm{~cm}^{-1}$ region is arising from the silicon substrate.

Polystyrene microparticles labelled with different fluorescent molecules (from Sigma Aldrich and Spherotech) were dissolved in water and dripped onto the periodic structure.

Particles having size ( $2 \mu \mathrm{m}$ diameter) similar to the period of the 3D structure were entrapped on the surface after washing. The lateral distribution of the beads was characterized by fluorescent microscopy as presented in Figure 3. The fluorescent molecules were analysed by SERS utilizing the plasmonic enhancement by the structured surface of the traps. The definite and sensitive differentiation of the molecules immobilized on the polystyrene bead surfaces are presented in Figure 4, where a huge increase in the Raman signal can be observed on the SERS surface. Taking into account that the Raman signal recorded from the beads on silicon substrate arises from the total volume of the particles, while SERS occurs only in the few nanometer region close to the SERS substrates, it can be concluded that the observed SERS enhancement of two orders of magnitude is much higher actually.
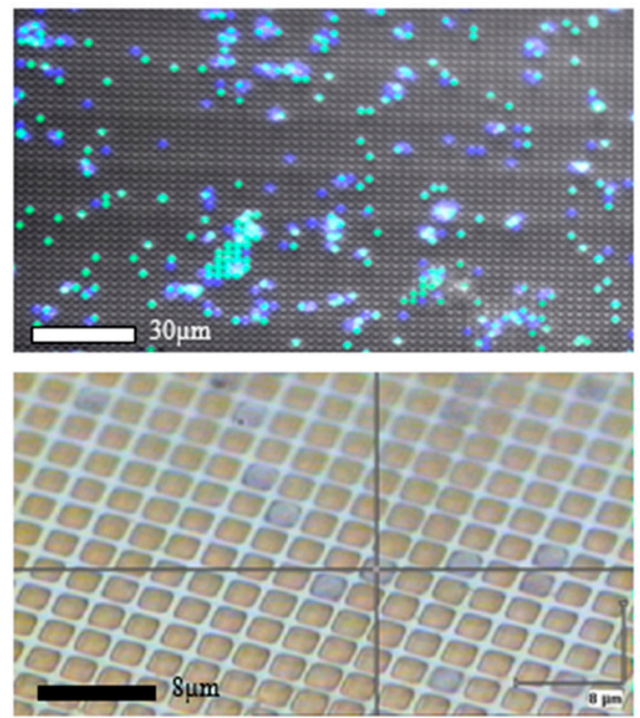

Figure 3. Fluorescent beads with appropriate $2 \mu \mathrm{m}$ diameter (Sigma Aldrich-green and Spherotech-blue) entrapped in the periodic array of perforated pyramidal structures: multichannel fluorescent image (top) and upright optical micrograph (bottom). 


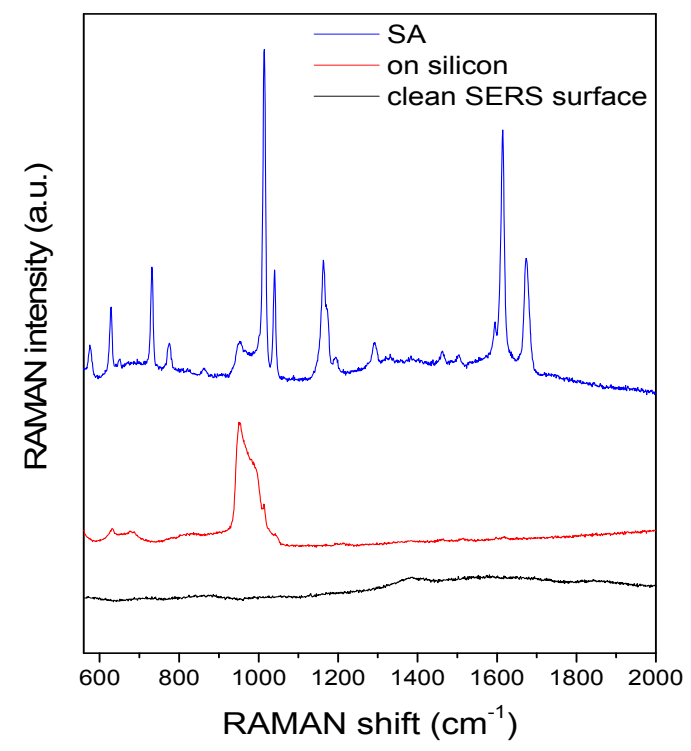

(a)

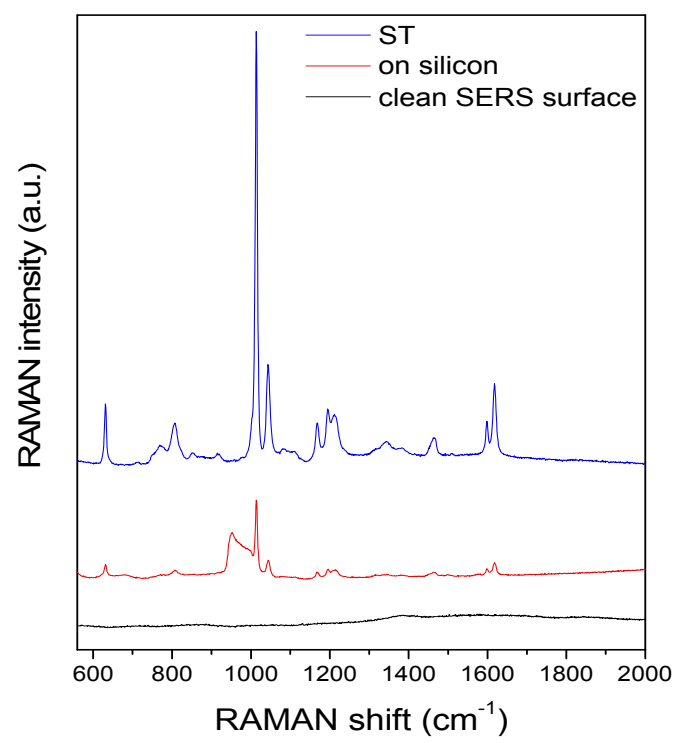

(b)

Figure 4. Comparison of the SERS spectra recorded on the clean SERS substrate (black) and different fluorescent beads on silicon (red) and trapped in the periodic array (blue). SA-Sigma Aldrich (a) and ST - Spherotech (b) fluorescent beads, respectively. The broad band in the $900-1000 \mathrm{~cm}^{-1}$ region is arising from the silicon substrate.

\section{Conclusions}

The applicability of special perforated periodic 3D structure was demonstrated for simultaneous particle (or cell) trapping and extremely sensitive Surface-Enhanced Raman Spectroscopy based detection of molecules immobilized on the surfaces of the confined beads.

Acknowledgments: This work was supported by the Hungarian Academy of Sciences. The authors gratefully acknowledge the high-quality technical work of Magda Erős and Margit Payer regarding microfabrication. Miklos Veres is grateful for the support of the János Bolyai Research Scholarship of the Hungarian Academy of Sciences.

Conflicts of Interest: The authors declare no conflict of interest. The founding sponsors had no role in the design of the study; in the collection, analyses, or interpretation of data; in the writing of the manuscript, and in the decision to publish the results. 


\section{References}

1. Ryder, A.G. Surface enhanced Raman scattering for narcotic detection and applications to chemical biology. Curr. Opin. Chem. Biol. 2005, 9, 489-493.

2. $\quad$ Etchegoin, P.; Maher, R.C.; Cohen, L.F.; Hartigan, H.; Brown, R.J.C.; Milton, M.J.T.; Gallop, J.C. New limits in ultrasensitive trace detection by surface enhanced Raman scattering (SERS). Chem. Phys. Lett. 2003, 375, 84-90.

3. Acuna, G.P.; Bucher, M.; Stein, I.H.; Steinhauer, C.; Kuzyk, A.; Holzmeister, P.; Schreiber, R.; Moroz, A.; Stefani, F.D.; Liedl, T.; et al. Distance Dependence of Single-Fluorophore Quenching by Gold Nanoparticles Studied on DNA Origami. ACS NANO 2012, 6, 3189-3195.

(C) 2017 by the authors. Licensee MDPI, Basel, Switzerland. This article is an open access article distributed under the terms and conditions of the Creative Commons Attribution (CC BY) license (http://creativecommons.org/licenses/by/4.0/). 